\title{
Microestrutura da fibra alimentar do albedo de laranja: um estudo por técnicas físicas e análise de imagens
}

\author{
Microstructure of dietary fiber from orange albedo: a study by microscopy and physical techniques
}

Maristela de Fátima Simplicio de SANTANA ${ }^{1 *}$, Carlos Alberto GASPARETTO²

\section{Resumo}

As fibras alimentares são consumidas naturalmente em cereais, frutas e hortaliças, mas também são adicionadas na forma concentrada em alimentos processados. Diferentes fontes de fibras têm diferentes estruturas e composições químicas, que irão definir a sua finalidade nutricional ou tecnológica. Este trabalho tem como objetivo a caracterização de fibra alimentar do albedo da laranja submetida a dois métodos de secagem (liofilização e convencional) e divididas por intervalos granulométricos. Para as determinações de densidade, área superficial, porosidade e distribuição de tamanho de poro, utilizaram-se técnicas clássicas da tecnologia de sistema particulado e foram comparadas com imagens de microscopia eletrônica de varredura. Os resultados apontaram que o método de secagem é o que mais influenciou na redução do volume de poros do material, também visualizado nas características microestruturais reveladas pela análise de imagem. O intervalo granulométrico da fibra particulada apresentou-se inversamente proporcional à densidade e à área superficial específica do material. A fibra alimentar do albedo da laranja apresentou características estruturais que permitem a diversificação de novos produtos alimentícios com alto valor nutritivo e comercial.

Palavras-chave: resíduos agroindustriais; tamanho de partículas; Citrus sinensis; propriedades físicas.

\begin{abstract}
The food fibers are consumed naturally in cereals, fruits and vegetables, and they are also added in a concentrated form in processed foods. Different fiber sources have different structures and chemical composition that will define their nutritional or technological purpose. This research aims the characterization of orange albedo dietary fiber submitted to two methods of drying (conventional and lyophilization) and split by granulometric intervals. For the density determinations, surface area, porosity and pore size distribution it was used classical techniques of the particulate systems tecnology and it was compared whit images of scanning electron microscopy. The results showed that the drying method influence more in the material pore volume reduction, this is also visualized in the microstructural characteristics reveled by image analysis. The granulometric interval of particulate fiber showed itself inversely proportional to the material density and material specific surface area. The orange albedo dietary fiber showed structural characteristics that allow the diversification of new food products with high nutritional and commercial value.
\end{abstract}

Keywords: agroindustrial waste; particles size; Citrus sp.; physical properties.

\section{Introdução}

Nos últimos anos tem havido um grande interesse em consumir alimentos contendo fibras alimentares, devido ao seu grande potencial de efeitos benéficos no trato intestinal e na prevenção de doenças degenerativas e crônicas (GUILLON; CHAMP, 2000). Este conhecimento tem resultado numa procura de novas fontes e de pesquisas voltadas para investigar a composição química, as propriedades nutricionais e tecnológicas, que são consequência de sua origem e das mudanças microestruturais sofridas pelo processamento ao qual foi submetido (THIMBAUD; LAHAYE; GUILLON, 1992; LARRAURI, 1999; GUILLON; CHAMP, 2000; SANGNARK; NOOMHORM, 2003; SANTANA, 2005).

As fontes naturais de fibra são frutas, raízes, legumes, tubérculos, grãos e cereais, mas pode-se encontrar no comércio, alimentos enriquecidos com estes nutrientes na forma concentrada, que são obtidos de resíduos da indústria alimentícia (ARUOMA, 1994; THASSITOU; ARVANITOYANNIS, 2001; PÉREZ; SANCHES, 2001), como exemplo, o processo industrial de suco de laranja, que utiliza como ração animal aproximadamente $50 \%$ do peso da fruta, material este que possui grande potencial como fonte de fibra (SANTANA, 2005).

No entanto, como se tem grande interesse nesse material, principalmente estando na forma de particulado, devido a sua maior aceitabilidade e facilidade de incorporação no processamento de produtos tradicionais (GRIGELMO-MIGUEL; MARTIN-BELLOSO, 1999), neste trabalho, procurou-se caracterizar a microestrutura da fibra alimentar do albedo de

${ }^{1}$ Laboratório de Agroindústria, Embrapa Amazônia Oriental, Trav. Dr. Enéas Pinheiro, s/n, CEP 66095-100, Belém - PA, Brasil, E-mail: msantana@cpatu.embrapa.br

${ }^{2}$ Laboratório de Propriedades Físicas, Departamento de Engenharia de Alimentos, Faculdade de Engenharia de Alimentos, Universidade Estadual de Campinas, Rua Monteiro Lobato, 80, Cidade Universitária, Barão Geraldo, CEP 13035-388, Campinas - SP, E-mail: gasparet@fea.unicamp.br

${ }^{*}$ A quem a correspondência deve ser enviada 
laranja, comparando-se o método de secagem convencional e por liofilização e diferentes intervalos granulométricos.

Várias técnicas são usadas para caracterizar a estrutura de alimentos, entretanto, algumas destas são usadas para obter dados específicos e quantitativos da estrutura interna de alimentos particulados, tais como: a análise por imagem e técnicas físico-químicas que são clássicas da tecnologia de partícula (SHOONMAN et al. 2001). Entre as características mais importantes de materiais particulados está sua densidade real e bulk, a porosidade e distribuição de tamanho de poro e sua área superficial específica (WEBB; ORR, 2001; JUSZCZAK; FORTUNA; WODNICKA, 2002).

\section{Material e métodos}

Neste trabalho, foram avaliadas fibras alimentares de albedo de laranja obtidas segundo metodologia descrita por Larrauri (1999). A secagem do material se deu em liofilizador, por aproximadamente 90 horas, e pelo método de secagem convencional com temperatura de $75^{\circ} \mathrm{C}$ e velocidade do ar de $1 \mathrm{~m} . \mathrm{s}^{-1}$. Depois, procedeu-se à separação em intervalos granulométricos, com peneiras em agitador mecânico, obtendo-se amostras descritas na Tabela 1 , com sua nomenclatura.

\subsection{Amostragem}

A amostragem do material foi efetuada a partir de uma massa de pó com 50 g, pelo método de quarteio, obtendo-se uma amostra representativa de $1 \mathrm{~g}$, em triplicata, para secagem em estufa a vácuo com temperatura de $40^{\circ} \mathrm{C}$ por 48 horas. Após secagem, as cápsulas com amostras foram acondicionadas em dessecador. O teor de umidade das amostras variou entre $4 \mathrm{e}$ $6 \%$ para as análises.

\subsection{Densidade real por picnometria}

O equipamento utilizado para determinação da densidade real das fibras foi o picnômetro a gás Hélio (MICROMERITICS ${ }^{\circledR}$, modelo Accupyc 1330). A metodologia utilizada foi de deslocamento de gás e a determinação experimental da densidade foi dividida em três etapas. Iniciou-se com a calibração do equipamento utilizando-se esferas de aço inox de volume precisamente $6,371710 \mathrm{~cm}^{3}$. Na calibração, foram utilizados os seguintes parâmetros: 19,85 psig para pressão exercida na

Tabela 1. Identificação das amostras de albedo.

\begin{tabular}{cccc}
\hline Amostras & Tipo de secagem & $\begin{array}{c}\text { Tamanho da } \\
\text { partícula }(\mathrm{mm})\end{array}$ & $\begin{array}{c}\text { Malha utilizada } \\
\text { (mesh) }\end{array}$ \\
\hline AL S 1 & Secador convencional & 0,425 a 0,300 & $-40+50$ \\
AL S 2 & Secador convencional & 0,300 a 0,210 & $-50+70$ \\
AL S 3 & Secador convencional & 0,210 a 0,149 & $-70+100$ \\
AL L 1 & Liofilizador & 0,425 a 0,300 & $-40+50$ \\
AL L 2 & Liofilizador & 0,300 a 0,210 & $-50+70$ \\
AL L 3 & Liofilizador & 0,210 a 0,149 & $-70+100$ \\
\hline
\end{tabular}

$\mathrm{AL}$ = albedo de laranja; $\mathrm{S}$ = secagem convencional; $\mathrm{L}$ = secagem em liofilizador; 1,2 e 3 tamanhos de partículas. câmara de amostra e de 0,001 psi.min ${ }^{-1}$ para taxa de equilíbrio. A temperatura medida foi de $27,0 \pm 1{ }^{\circ} \mathrm{C}$.

O material foi pesado, colocado na câmara para realização das 20 purgas e iniciadas as leituras. A precisão adotada para os resultados foi de $0,01 \%$ e o número de corridas igual a 10 , parâmetro estabelecido em ensaios preliminares. Os valores das taxas de equilíbrio testadas variaram entre $5 \times 10^{-4}$ psi.min ${ }^{-1}$; $5 \times 10^{-3}$ psi.min ${ }^{-1} ; 5 \times 10^{-2}$ psi.min ${ }^{-1}$, com número de purgas igual a 10. Os melhores resultados apresentaram-se na taxa de equilíbrio de $5 \times 10^{-3}$ psi.min . $^{-1}$.

\subsection{Densidade bulk e real por porosimetria de mercúrio}

O aparelho utilizado na caracterização das amostras foi o AutoPore III fabricado pela MICROMERITICS ${ }^{\circledR}$, o qual pode detectar poros de até $6 \mathrm{~nm}$ como limite inferior. Para a realização da análise, foi escolhido o penetrômetro s/n - (10)\% Bulb, 1.131 Stem, Powder, a análise no porosímetro consistiu de duas etapas à baixa e à alta pressão, respectivamente $(0,5$ até 30000 psi). Este utiliza uma tensão superficial do Mercúrio de 485 din. $\mathrm{cm}^{-1}$. Os parâmetros estabelecidos para análise na baixa pressão foram: tempo de equilíbrio de 60 segundos após cada aumento de pressão, pressão de evacuação de $50 \mu \mathrm{mHg}$, pressão de preenchimento de Mercúrio de 10 psia. Para análise na alta pressão, foi utilizado tempo de equilíbrio de 30 segundos e volume máximo de intrusão de $100 \mathrm{~cm}^{3} \cdot \mathrm{g}^{-1}$.

A densidade bulk requer o valor de volume total da amostra antes do preenchimento com Mercúrio, que é fornecida ao software. É obtida dividindo o peso da amostra por este volume. O volume da amostra é encontrado subtraindo-se do volume do penetrômetro vazio e o volume de Mercúrio que ocupa o espaço vazio não ocupado pela amostra. A determinação do volume exige a medida da massa do penetrômetro vazio, $M_{p}$, o peso da amostra $M_{s}$ e o peso total $M_{\text {total }}$ do penetrômetro preenchido com Mercúrio, isto é (Equação 1):

$V_{H g}=\frac{M_{H g}}{\rho_{H g}}=\frac{M_{t o t a l}-M_{p}-M_{s}}{\rho_{H g}}$

Se Vp é o volume do penetrômetro vazio, o volume bulk da amostra $\mathrm{V}_{\text {bulk }}$ é o volume do penetrômetro menos o volume de Mercúrio $\mathrm{V}_{\mathrm{Hg}}$. A densidade bulk, $\rho_{\text {bulk' }}$, da amostra é então obtida por (Equação 2):

$\rho_{b u l k}=\frac{M_{s}}{V_{p}-V_{\mathrm{Hg}}}$

O volume absoluto da amostra é encontrado subtraindo o volume de Mercúrio que penetra os poros do material (V), obtido no teste de alta pressão, do volume bulk. Assim, a densidade real da matriz sólida $\rho_{\mathrm{r}}$ é obtida (Equação 3):

$\rho_{r}=\frac{M_{s}}{V_{b u l k}-V}$ 
A porosidade da amostra é obtida pela Equação 4:

$\varepsilon=1-\frac{\rho_{\text {bulk }}}{\rho_{r}}$

Para efeito de comparação entre os métodos, foi calculada a porosidade, também pela Equação 4, utilizando-se a densidade real obtida pelo picnômetro.

\subsection{Obtenção da isoterma de adsorção de nitrogênio}

Foram determinadas as medidas de área superficial específica, volume de microporo e diâmetro médio de poro usando o equipamento Micromeritics Gemini 2375 V4.01. O método empregado foi o de adsorção de nitrogênio gasoso em condições criogênicas $\left(\mathrm{T}=-196^{\circ} \mathrm{C}\right)$, utilizando banho de nitrogênio líquido de alta pureza. $\mathrm{O}$ equipamento apresenta as seguintes especificações: área superficial específica $\geq 0,001 \mathrm{~m}^{2} \cdot \mathrm{g}^{-1} \mathrm{e}$ total $\geq 0,1 \mathrm{~m}^{2} \cdot \mathrm{g}^{-1}$; volume de poro $\geq 4 \times 10^{-6} \mathrm{~cm}^{3} \cdot \mathrm{g}^{-1}$; faixa de pressão relativa: adsorção $=0,1$ a $1,0 \mathrm{P} / \mathrm{P}_{\mathrm{o}}$ e dessorção $=1,0 \mathrm{a}$ $0,1 \mathrm{P} / \mathrm{P}_{\mathrm{o}}$; faixa de medida de pressão $=0$ a $950 \mathrm{mmHg}$; pressão de resolução $<0,1 \mathrm{mmHg}$; precisão e linearidade $= \pm 0,5 \%$; resolução mínima de pressão relativa $\left(\mathrm{P} / \mathrm{P}_{\mathrm{o}}\right)<10^{-4}$. Os parâmetros de operação informados ao programa do equipamento foram os seguintes: taxa de evacuação de 100,00 mmHg/min, o tempo de evacuação de 5,0 minutos, a pressão de saturação de 713,30 mmHg, tempo de equilíbrio de 10 segundos, modelo de análise por equilíbrio e massa da amostra.

\section{5 Área superficial específica}

Obtida a isoterma de adsorção, o software do equipamento calcula a área superficial específica multiponto pela teoria de BET (BRUNNAUER, EMMETT e TELLER, 1938) descrita segundo Webb e Orr (1997), adotando a relação linearizada pela curva de $\mathrm{P} / \mathrm{V}_{\mathrm{m}}\left(\mathrm{P}_{\mathrm{o}}-\mathrm{P}\right)$ em função de $\mathrm{P} / \mathrm{P}_{\mathrm{o}}$, cuja inclinação é obtida pela Equação 5:

$s=\frac{C-1}{V_{m} C}$

e intersecção (Equação 6)

$i=\frac{1}{V_{m} C}$

O volume da monocamada de gás adsorvido $\mathrm{V}_{\mathrm{m}}$ e a constante $\mathrm{C}$ de BET são calculados a partir da inclinação e da intersecção (Equação 7 e 8):

$V_{m}=\frac{1}{s+i}$

$C=\frac{s}{i}+1$

$A$ área superficial específica, $\mathrm{S}_{\mathrm{BET}}\left(\mathrm{m}^{2} \cdot \mathrm{g}^{-1}\right)$, da amostra é calculada por (Equação 9):
$S_{B E T}=\frac{V_{m} \sigma N_{A}}{m V_{o}}$

onde $\sigma$ é a área efetiva ocupada por uma molécula de adsorbato (1,6 x 10-20 $\mathrm{m}^{2}$ para o nitrogênio); N é o número de Avogadro $\left(\mathrm{mol}^{-1}\right)$; $\mathrm{m}$ a massa de adsorbato $(\mathrm{g})$; e Vo é o volume molar do gás a STP $\left(\mathrm{cm}^{3} \cdot \mathrm{mol}^{-1}\right)$.

\subsection{Volume e diâmetro de poro}

$\mathrm{O}$ volume acumulativo de poro $\left(\mathrm{cm}^{3} \cdot \mathrm{g}^{-1}\right)$ e o diâmetro médio do poro $(\mathrm{nm})$ foram determinados a uma pressão relativa de 0,99 .

\subsection{Distribuição de tamanho de poro}

A distribuição de tamanho de poro foi obtida aplicando-se o método BJH (BARRET; JOYNER; HALENDA, 1951). Este método é baseado no modelo de condensação capilar desenvolvido por Cohan (1938), o qual oferece a existência de uma camada adsorvida na parede do poro preenchido com o adsorbato condensado e aquele esvaziado. De acordo com Cohan, 1938 a espessura da camada é dada pela temperatura em função da pressão relativa, não dependendo do raio do poro. Em todos os métodos baseados na condensação capilar, o volume do poro esvaziado, na mudança de pressão em um certo estágio do processo de adsorção, é função da quantidade de adsorbato adsorvido naquele estágio e calculado como volume líquido, conforme Equação 10.

$\Delta V^{x}=R_{i}\left(\Delta v_{i}-B_{i}\right)$

onde $V^{x}$ é o volume de mesoporos vazios dado pela variação de pressão no estágio de dessorção; $\mathrm{V}_{\mathrm{i}}$ é o volume do líquido adsorvido; $\mathrm{R}_{\mathrm{i}}$ é o coeficiente adimensional expressando a transição do volume de líquido de adsorbato que evaporou dos poros (esvaziados) em um certo estágio, para o volume de poro em que aconteceu a dessorção; $\mathrm{B}_{\mathrm{i}}$ é o termo de correção que expressa a redução da camada de adsorção do líquido ainda presente no poro esvaziado durante o estágio anterior de dessorção.

Os cálculos são feitos com base na isoterma de adsorção usando os seguintes dados: pressão relativa e volume de adsorbato correspondente e a espessura da camada adsorvida. De acordo com os autores do método, durante o primeiro estágio de dessorção a camada de adsorção é reduzida por $\Delta$ ti no poro vazio deste e de estágios anteriores. Esta consideração é justificada por ser o uso do coeficiente $\mathrm{R}_{\mathrm{i}}$ e o termo de correção $\mathrm{B}_{\mathrm{i}}$.

A relevante fórmula pode finalmente ser descrita como (Equação 11):

$\Delta V i^{x}=\frac{r_{i}^{2}}{\left(r_{T_{i}}+\Delta t_{i}\right)^{2}}\left(\Delta V_{i}-\Delta t_{i} \sum_{j=1}^{j=i-1} \frac{r_{j}-t_{j}}{r_{j}} \Delta S_{j}^{x}\right)$

onde $\Delta \mathrm{V}_{\mathrm{i}}$ é o volume de mesoporos esvaziados em dada pressão relativa em certo estágio da dessorção; $\Delta \mathrm{V}_{\mathrm{i}} \mathrm{o}$ volume líquido do adsorbato; $\mathrm{r}_{\mathrm{i}}$ o raio médio efetivo do poro esvaziado durante $\mathrm{o}$ i $\mathrm{e}$ 
j estágio de dessorção e adsorção, respectivamente; $\mathrm{rt}_{\mathrm{i}}$ é a média Kelvin no centro do raio do poro esvaziado durante o estágio i; $\Delta t_{i}$ é a espessura de adsorção da camada que restou no poro; $\mathrm{r}_{\mathrm{j}}$ o raio médio efetivo do poro esvaziado durante o j estágio de adsorção; $t_{j}$ espessura da camada adsorvida presente no poro; $\mathrm{S}^{\mathrm{x}}$ é a área superficial do poro esvaziado de adsorbato durante o processo de dessorção.

No método BJH, a superfície da camada adsorvida nos poros esvaziados durante estágios subsequentes de dessorção é o produto da área superficial do poro e da proporção da média de Kelvin (que relaciona a curvatura do menisco em um poro cilíndrico à pressão relativa na qual a condensação ocorre) no centro do raio do poro esvaziado no estágio i. A superfície cumulativa, volume de poro e diâmetro médio do poro são obtidos com base na isoterma de adsorção de 1,7 a $30 \mathrm{~nm}$.

\subsection{Microscopia eletrônica de varredura}

A estrutura da fibra alimentar dos materiais estudados foi observada utilizando-se imagens de microscopia eletrônica de varredura (MEV) geradas no equipamento JSM 5900 LV do Laboratório Nacional de Luz Sincrotron (LNLS/MCT) em Campinas, SP. A tensão usada foi de $15 \mathrm{kV}$, em diversas magnitudes e diâmetro do feixe de $25 \mathrm{~mm}$ e detector de imagens elétrons secundários.

\section{Resultados e discussão}

\subsection{Densidade, porosidade e área superficial}

\section{Densidade}

Os resultados experimentais para densidade real determinados pelo picnômetro e porosímetro e a densidade bulk das fibras são apresentados na Tabela 2 . O desvio padrão dos resultados da densidade real do picnômetro é da ordem de grandeza de $10^{-2}$, comprovando que as análises realizadas no picnômetro de gás Hélio apresentaram boa reprodutibilidade.

Os valores de densidade real (picnômetro) da fibra alimentar do albedo de laranja não diferiram com relação ao método de secagem. Os maiores valores obtidos foram para fibra alimentar da amostra liofilizada (amostra AL L), em torno de 1,6 g. $\mathrm{cm}^{-3}$. Quanto à diferença de tamanho das fibras, foi possível estabele- cer uma relação inversamente proporcional, estando de acordo com a densidade da maltodextrina, 1,27 g. $\mathrm{cm}^{-3}$ determinado pelo método de picnometria a gás Hélio por Collares (2002), e diferiram dos resultados para casca de laranja liofilizada, de 9,97 g. $\mathrm{cm}^{-3}$ determinada em porosimetria de Mercúrio por Panchev e Karageorgiev (2000).

Nos resultados experimentais, para densidade bulk obtida por porosimetria de Mercúrio das fibras, verifica-se que os valores para esta, foram de duas a três vezes menores quando o material foi desidratado por liofilização com relação ao lote submetido à secagem convencional.

$\mathrm{O}$ volume esqueleto da amostra pode ser determinado com a entrada de Mercúrio nos poros através do incremento de pressão. Quando na pressão máxima todos os poros abertos estiverem preenchidos, o volume de Mercúrio introduzido na amostra é igual ao volume total de poros (WEBB, 2001a). A diferença entre o volume total de poros e o volume envelope ou bulk, é o volume real quando a amostra não tiver poros fechados. Se a amostra contém poros menores que o diâmetro mínimo de poro em que o Mercúrio pode ser introduzido $(3 \mathrm{~nm})$, a precisão na determinação do volume (esqueleto) está comprometida. Nestes materiais, o volume real deveria ser menor que o obtido na picnometria. Gases como Hélio e Nitrogênio podem penetrar em microporos e mesoporos onde o Mercúrio não pode ser introduzido. A diferença entre o volume esqueleto obtido pela porosimetria e o volume real obtido pela picnometria indica uma limitação do método porque a molécula de Mercúrio não preenche um certo volume dos poros existentes que possuem tamanho mínimo que são preenchidos pela molécula de hélio. A densidade real do porosímetro de Mercúrio é comparada ao do picnômetro como medida da veracidade dos poros que o equipamento está analisando. De acordo com a técnica, é esperado que esta seja maior devido ao tamanho da molécula de Mercúrio ser maior do que a do Hélio. A diferença no volume obtido através da porosimetria de Mercúrio e aquele obtido na picnometria indica uma faixa de volume do poro mínimo que é correspondente ao tamanho da molécula do gás; esta observação encontra-se de acordo com Webb (2001b).

\section{Porosidade}

Os resultados médios para a porosidade total, a calculada pela Equação 4 e a determinada pelo porosímetro de Mercúrio,

Tabela 2. Resultados de densidade real e bulk de fibra alimentar.

\begin{tabular}{lcccccc}
\hline & AL L 1 & AL L 2 & AL L 3 & AL S 1 & AL S 2 & AL S 3 \\
\hline Densidade real (picnômetro) & 1,549 & 1,568 & 1,610 & 1,514 & 1,519 & 1,520 \\
Desvio padrão & 0,004 & 0,019 & 0,001 & 0,000 & 0,000 & 0,001 \\
Densidade bulk (porosímetro) & 0,633 & 0,461 & 0,438 & 1,221 & 0,147 & 0,004 \\
Desvio padrão & 0,003 & 0,000 & 0,009 & 0,002 & 2,041 & 0,006 \\
Densidade real (porosímetro) & 2,175 & 1,060 & 1,127 & 1,780 & 1,692 \\
Porosidade equação 4 & 59,11 & 70,58 & 72,77 & 19,36 & 351 & 43,81 \\
Porosímetro de Hg & 70,88 & 56,50 & 61,09 & 31,42 & 41,65 \\
Área superficial específica S $_{\text {BET }}$ & 2,2447 & 2,3016 & 1,6491 & 0,8248 & 1,0338 & 1,1989 \\
\hline
\end{tabular}

$\mathrm{AL}$ = albedo de laranja; $\mathrm{S}$ = secagem convencional; $\mathrm{L}$ = secagem em liofilizador; 1, 2 e 3 tamanhos de partículas. 
são apresentados na Tabela 2. A diferença entre os resultados obtidos pela Equação 4 e os obtidos pelo porosímetro de Mercúrio se deve ao fato de que o porosímetro utiliza a mesma equação para o cálculo da porosidade, mas usando o valor de densidade real obtido de uma maneira mais precisa pelo picnômetro, devido à maior capacidade do gás Hélio em penetrar os microporos dos materiais, e, ainda, com a utilização da Equação 4 que corrige o volume da massa de sólido obtido pelo picnômetro, sempre existirá uma diferença entre estas duas formas de obtenção da porosidade. Quando se utiliza a densidade real fornecida pelo picnômetro, o resultado obtido para a porosidade é menos sujeito a erros e mais confiável do que aquele fornecido pelo porosímetro, particularmente no caso de microporos, devido à limitação do porosímetro em detectar este intervalo de poros.

Panchev e Karageorgiev (2000) encontraram valores de $31,40 \%$ de porosidade para casca de laranja liofilizada, valor este bem abaixo dos encontrados para fibra de albedo da laranja neste trabalho, com exceção para a amostra AL S 1.

\section{Área superficial}

Os resultados de área superficial, obtidos a partir dos dados de adsorção de Nitrogênio calculados pelo método BET para as fibras obtidas de albedo de laranja, são apresentados na Tabela 2. Comparando-se os métodos de secagem, verificase que as fibras liofilizadas apresentaram valores maiores de área superficial específica. Este resultado está de acordo com a literatura sobre materiais liofilizados que, por constituir um método de secagem em vácuo intenso, promovendo a sublimação do gelo formado, preserva a integridade física do material e garante as propriedades originais do produto (LIAPIS, 1987). Comparando-se a mesma granulometria e diferentes métodos de secagem, verifica-se que as amostras liofilizadas obtiveram mais que o dobro da área superficial da fibra seca em bandeja. Isto indica que o processamento ao qual foi submetido (secagem convencional com temperatura de $75^{\circ}$ e velocidade de ar de $1 \mathrm{~m} \cdot \mathrm{s}^{-1}$ ) promoveu uma considerável redução de área superficial específica da fibra, sendo necessário avaliar estes materiais em um método de secagem mais brando, sem perder de vista a viabilidade econômica.

A área superficial específica, para a maioria das amostras, apresentou-se crescente com relação ao tamanho de partícula, estando de acordo com a literatura. Este comportamento asse- gura grande diferença na adsorção de diversas substâncias como água, óleo, e outros, e nas reações, quando adicionar partículas de fibra em alimentos. O conhecimento desse comportamento é indispensável nos cálculos e definições dos processos de engenharia. É necessário ressaltar que a fibra alimentar do albedo da laranja é um material orgânico, sem forma geométrica definida.

Segundo Svarovsky (1987), a forma da partícula afeta a interação entre sólido poroso e o gás, partículas como pós, flocos e materiais fibrosos apresentam certa obstrução do fluxo, devido ao arranjamento de partículas, que promove maior área de contato entre partículas menores, assim, a área superficial obtida com métodos de interação como a adsorção de gás apresentam-se menores.

Comparando-se estes dados com outros trabalhos com materiais biológicos, verificamos que as fibras possuem área superficial específica próxima às da maltodextrina (Schoonman et al., 2001), encontrando um intervalo de $0,01 \mathrm{a} 1,52 \mathrm{~m}^{2} \cdot \mathrm{g}^{-1}$. Berlin, Howard e Pollansk (1964) determinaram a área superficial de leite em pó, pela teoria BET em isoterma de nitrogênio, em diferentes métodos de secagem, encontrando valores de 0,10 a $0,64 \mathrm{~m}^{2} \cdot \mathrm{g}^{-1}$. A área superficial total da palha e de frações do grão de trigo para uso como fibra alimentar, foi determinada por Chesson, Gardner e Wood (1997) que, usando o método de adsorção de gás, encontraram para internódio $3,3 \mathrm{~m}^{2} \cdot \mathrm{g}^{-1}$; totalidade do grão, $0,7 \mathrm{~m}^{2} \cdot \mathrm{g}^{-1}$; grão moído, $1,0 \mathrm{~m}^{2} \cdot \mathrm{g}^{-1}$; fração do farelo, $1,1 \mathrm{~m}^{2} \cdot \mathrm{g}^{-1}$; fração aleurone, $1,5 \mathrm{~m}^{2} \cdot \mathrm{g}^{-1}$; e para fração do endosperma, $1,6 \mathrm{~m}^{2} \cdot \mathrm{g}^{-1}$.

\subsection{Distribuição de poro por adsorção de nitrogênio}

Na Tabela 3, observam-se os resultados da estimativa de porosidade por adsorção de Nitrogênio, calculados pelos métodos de BET e BJH. O volume de mesoporos das fibras submetidas ao processo de liofilização foi 2 a 3 vezes maior que para as fibras secas em bandejas, indicando que o processamento ao qual foi submetido promoveu danos aos poros.

Para a fibra alimentar de laranja submetida ao processo de secagem convencional, a superfície específica (Tabela 2) apresentou-se diretamente proporcional ao volume de mesoporos (Tabela 3), e apresentou-se inversamente proporcional ao tamanho da partícula. O volume de mesoporos, calculado

Tabela 3. Caracterização dos poros das fibras pelos métodos BET e BJH.

\begin{tabular}{cccccc}
\hline Amostra & $\begin{array}{c}\text { Volume de mesoporos } \\
\mathrm{cm}^{3} \cdot \mathrm{g}^{-1} \times \mathrm{10}^{3}\end{array}$ & $\begin{array}{c}\text { Diâmetro médio de } \\
\text { mesoporos nm }\end{array}$ & $\begin{array}{c}\text { Área superficial } \\
\text { acumulativo de poros } \mathrm{m}^{2} \cdot \mathrm{g}^{-1}\end{array}$ & $\begin{array}{c}\text { Volume acumulativo } \\
\text { de poros } \mathrm{cm}^{3} \cdot \mathrm{g}^{-1} \times 0^{3}\end{array}$ & $\begin{array}{c}\text { Diâmetro médio de } \\
\text { poros } \mathrm{nm}^{3}\end{array}$ \\
\hline AL L 1 & 3,00 & 6,122 & 1,235 & 1,812 & 9,823 \\
AL L 2 & 3,00 & 5,027 & 1,605 & 2,422 & 9,363 \\
AL L 3 & 2,20 & 5,312 & 1,120 & 1,322 & 9,814 \\
AL S 1 & 0,90 & 4,672 & 0,506 & 0,689 & 9,743 \\
AL S 2 & 1,10 & 4,212 & 0,603 & 0,759 & 10,174 \\
AL S 3 & 1,40 & 4,823 & 0,681 & 1,026 & 10,504 \\
\hline
\end{tabular}

$\mathrm{AL}=$ albedo de laranja; $\mathrm{S}=$ secagem convencional; $\mathrm{L}$ = secagem em liofilizador; 1,2 e 3 tamanhos de partículas. 
pelo método BET, mostrou-se próximo ao de $\mathrm{BJH}$ para poros acumulados.

As Figuras 1a e b apresentam as curvas de frequência de poro ou distribuição de tamanho de poro. Observa-se que o grupo de poro predominantemente encontra-se em 2 a $20 \mathrm{~nm}$ de diâmetro em todas as amostras estudadas, caracterizando-se, por esta análise, como um material mesoporoso.

Para o albedo de laranja liofilizado (Figura 1a), o tamanho 1, com partículas entre 0,30 e 0,42 mm (amostra ALL 1), obteve maior volume de poros com diâmetro entre 2 e $5 \mathrm{~nm}$. O tamanho 2, com partícula intermediária entre 2 e $3 \mathrm{~nm}$ e tamanho 3 (amostra ALL 2), com menores partículas, destacou-se dos demais com volume de poro próximo de $5 \mathrm{~cm}^{3} \cdot \mathrm{g}^{-1}$ com diâmetro
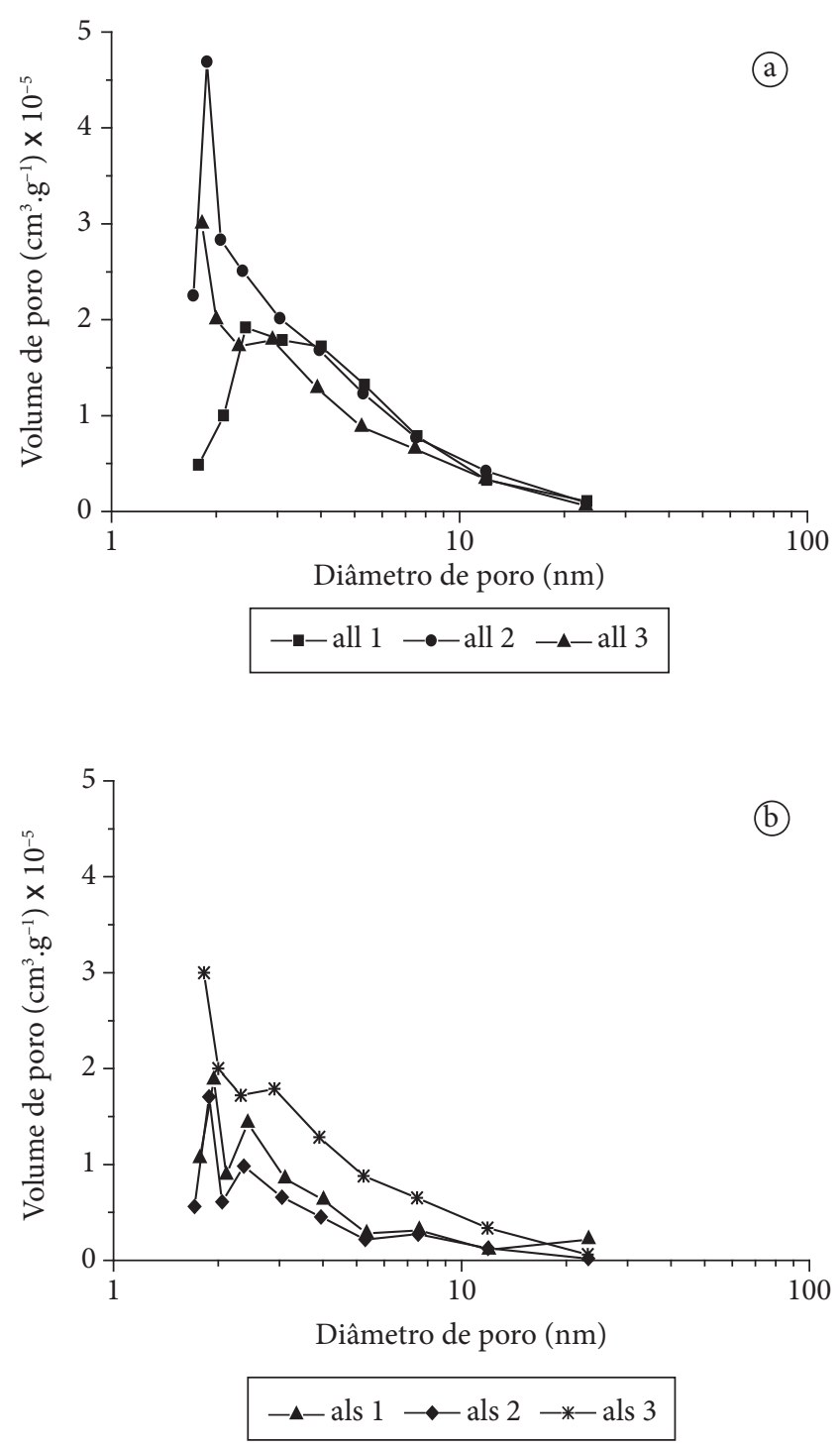

Figura 1. Distribuição de tamanho de poro $(\Delta \mathrm{Vp} / \Delta \mathrm{dp}$ versus $\mathrm{dp})$ para fibra alimentar de albedo de laranja obtida pelo método BJH com base na adsorção de nitrogênio. $\mathrm{AL}=$ albedo de laranja; $\mathrm{S}$ = secagem convencional; $\mathrm{L}$ = secagem em liofilizador; 1 , 2 e 3 tamanhos de partículas. de $2 \mathrm{~nm}$. No processo de secagem convencional, os tamanhos 1 e 2 (amostras ALL 1 e 2, maiores partículas) obtiveram 2 picos de volume com $2 \mathrm{~nm}$ e 2,5 nm, e o tamanho 3 (amostra ALL 3), com maior número de poros em $2 \mathrm{~nm}$. Comparandose os métodos de secagem, observa-se que o convencional em bandeja obteve valores 40\% menores que o liofilizado. Quanto à diferença de diâmetro, não foi possível estabelecer tendência de comportamento.

Os resultados apontam que o tratamento de secagem convencional promoveu danos consideráveis na microestrutura, possivelmente devido ao encolhimento dos poros, e, consequentemente, na qualidade das fibras quanto a suas propriedades funcionais fisiológicas e funcionais, que irão diferenciar seu uso e suas propriedades de engenharia como os cálculos de processo e design de máquinas.

As curvas de volume acumulativo de poro versus diâmetro de poro para fibra alimentar de albedo, são mostradas na Figura 2. O volume de poro acumulativo indica a quantidade de poro existente com um diâmetro respectivo. Observa-se que, para todas as amostras, um maior volume de poros encontra-se abaixo de $12 \mathrm{~nm}$ de diâmetro.

Verifica-se que as fibras de albedo de laranja liofilizadas apresentaram volume de poro acima das fibras desidratadas pelo método convencional. Portanto, possui diferenças entre os métodos de secagem, mas não revela tendência com a diferença de tamanho da partícula.

\subsection{Distribuição de tamanho de poro por porosimetria de mercúrio}

Os resumos dos relatórios fornecidos pela análise experimental das fibras com seus respectivos resultados estão

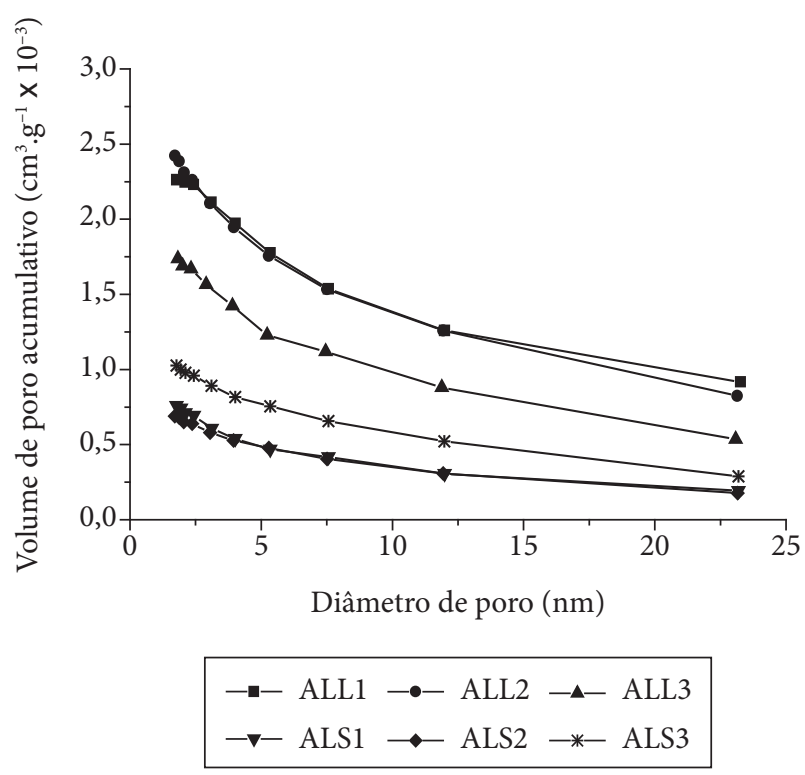

Figura 2. Distribuição de tamanho de poro por adsorção de nitrogênio para fibra alimentar. 
apresentados na Tabela 4. As análises apresentaram volume de intrusão de Mercúrio dentro da faixa ideal (de 25 a 90\%). Comparando-se os dados das amostras de casca de laranja obtidos por Panchev e Karageorgiev (2000), verifica-se que os valores de área superficial determinados neste trabalho para albedo de laranja apresentam-se maiores.

O gráfico mais básico da porosimetria é o volume de intrusão de Mercúrio em função do diâmetro, neste, a mudança na inclinação da curva e na sua forma geral oferece uma visão de como a porosidade é distribuída na amostra, como aconteceu o preenchimento, se ocorreu colapso da estrutura e indica se o material sofreu compressão com a aplicação de alta pressão.

Na Figura 3, observam-se as curvas de intrusão e extrusão em função do diâmetro de poro para as fibras de albedo de laranja liofilizado e desidratado em secador convencional com diferentes tamanhos de partículas. A histerese característica é mostrada e tem sido encontrada por outros sistemas de alimentos. $\mathrm{O}$ fenômeno de histerese pode ser explicado pela existência de poros com o formato similar a uma "garrafa com gargalo" que retêm o volume de Mercúrio injetado durante a extrusão devido à forma geométrica dos poros que dificultam o seu esvaziamento.

As curvas de distribuição de volume em função do diâmetro (Figura 4) apresentam uma diferença de volume injetado entre a fibra de albedo de laranja liofilizada e desidratada em secagem convencional. $\mathrm{O}$ volume injetado no material submetido à liofilização variou entre 1,0 e $1,6 \mathrm{~cm}^{3} \cdot \mathrm{g}^{-1} \mathrm{e}$, para o material desidratado em secador convencional, o volume máximo injetado foi de $0,4 \mathrm{~cm}^{3} \cdot \mathrm{g}^{-1}$.

Comparando-se os métodos de secagem, constata-se que, em geral, a fibra desidratada pelo método convencional apresentou redução de aproximadamente $90 \%$ no volume acumulativo. Quanto à diferença de tamanho não se verificaram alterações para a faixa estudada. A comparação dos métodos de secagem, para albedo de laranja, revelou que a secagem convencional apresentou diferença pouco significativa na redução da quantidade e no diâmetro dos poros. Em quase todas as amostras submetidas à desidratação em secador convencional, é possível observar que os picos apresentam-se nos menores diâmetros da faixa de distribuição.

A comparação entre a intrusão e extrusão mostra uma histerese pouco significativa. Comparando-se as curvas obtidas neste estudo e as apresentadas por Panchev e Karageorgiev (2000) para casca e polpa de maçã e casca de laranja, observa-se uma diferença considerável no comportamento: a histerese obtida pelos autores indicou que os tubos capilares com dimensões constantes não eram os principais elementos que constituem a estrutura de poros capilares. Alguns autores têm estudado o fenômeno das histereses obtidas pela porosimetria de Mercúrio e sugerido métodos para avaliar o uso da teoria da percolação, simulações de computador ou outras técnicas adicionando parâmetros como a forma do poro (tipo "gargalo"), distribuição de tamanho ao longo do corpo do poro, a forma da secção transversal do "gargalo" do poro e outros (IOANNIDIS; CHARTZ, 1993; LEE et al., 1994; MATTHEWS et al., 1995). Formas de poros podem ser observadas nas microfotografias obtidas por Microscopia Eletrônica de Varredura, apresentadas nas Figuras 5a-f, que revelam as irregularidades das estruturas físicas do material orgânico. Também é possível verificar diferenças nas estruturas quanto ao método de secagem ao qual foi submetido o material. Os efeitos do método de secagem convencional ( $\mathrm{Fi}$ gura 5d-f) revelam um encolhimento maior pouco significativo quando comparados com dados de densidade, mas, quando avaliados em distribuição de tamanho de poros, revelam uma diminuição de aproximadamente $90 \%$ no volume acumulativo. Tal observação, quando consideradas as possíveis reações em processos de transferência de calor e de velocidade de reações químicas, pode ser significativa.

Rouquerol, Rouqueirol e Sing (1999) comentam que materiais vegetais são classificados como "organização de substâncias que estão sob a ação da vida e onde vários processos físico-químicos complexos estão geralmente envolvidos" e, para estes, não são conhecidos os parâmetros da estrutura de poros capilares. Entretanto, pode-se utilizar a metodologia da porosimetria de Mercúrio como uma descrição qualitativa dos materiais.

\section{Conclusões}

De acordo com os dados obtidos neste estudo, é possível concluir que o método de secagem é o parâmetro que mais influenciou nas características microestruturais da fibra alimentar de albedo de laranja, observando-se uma significativa redução na porosidade da fibra desidratada em secador convencional, classificando o material como mesoporoso. O intervalo granulométrico não revelou tendência de comportamento para as características determinadas, com exceção para área superficial.

Tabela 4. Resultados das análises no porosímetro de mercúrio para as fibras alimentares.

\begin{tabular}{|c|c|c|c|c|c|c|}
\hline & \multirow{2}{*}{$\begin{array}{l}\text { Volume total de } \\
\text { intrusão }\end{array}$} & \multirow{2}{*}{ Área total de poro } & \multicolumn{3}{|c|}{ Diâmetro médio de poro } & \multirow{2}{*}{$\begin{array}{c}\text { Volume usado da } \\
\text { haste }\end{array}$} \\
\hline & & & Volume & Área & $4 \mathrm{~V} / \mathrm{A}$ & \\
\hline & $\mathrm{cm}^{3} \cdot \mathrm{g}^{-1}$ & $\mathrm{~m}^{2} \cdot \mathrm{g}^{-1}$ & & $\mathrm{~nm}$ & & $\%$ \\
\hline AL L 1 & 1,119 & 43,545 & $6.484,9$ & 9,50 & $1.028,0$ & 60,0 \\
\hline AL L 3 & 1,393 & 89,820 & $7.816,7$ & 8,50 & 620,0 & 39,0 \\
\hline AL S 1 & 0,257 & 19,160 & $5.017,7$ & 8,80 & 537,0 & 44,0 \\
\hline
\end{tabular}



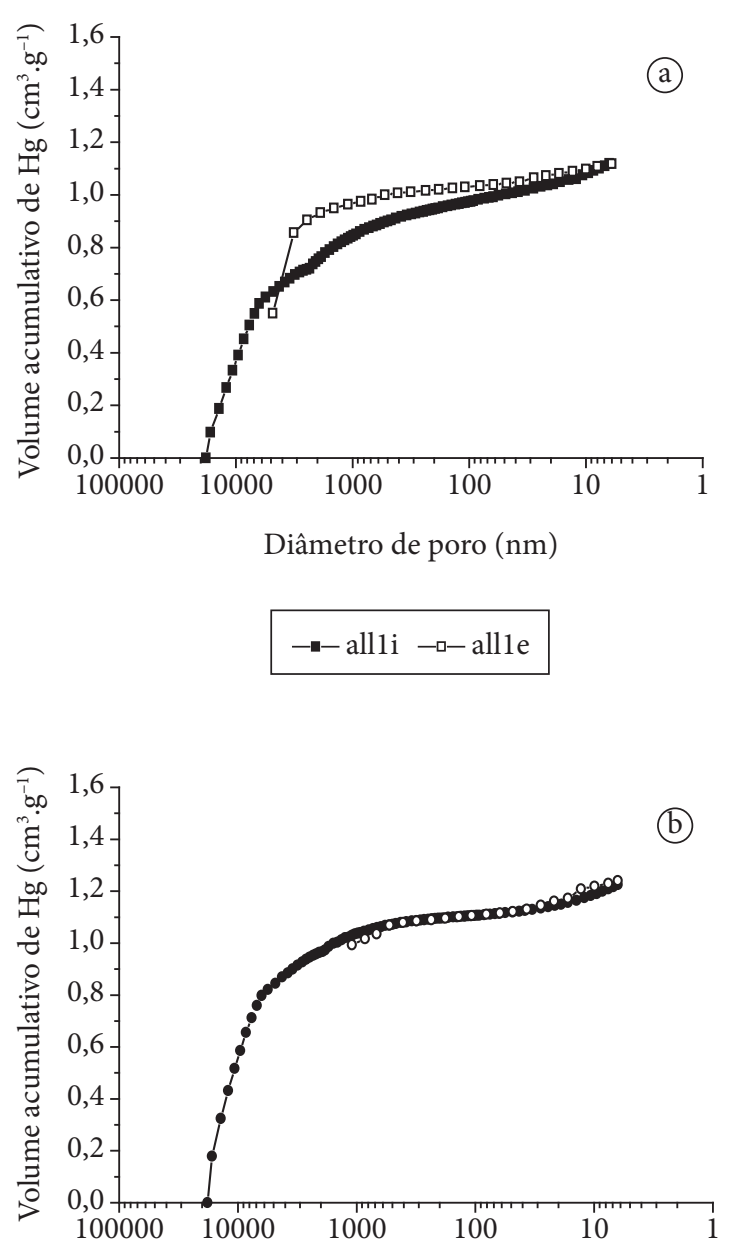

Diâmetro de poro (nm)

$$
\rightarrow \bullet \text { all2i -o- all2e }
$$

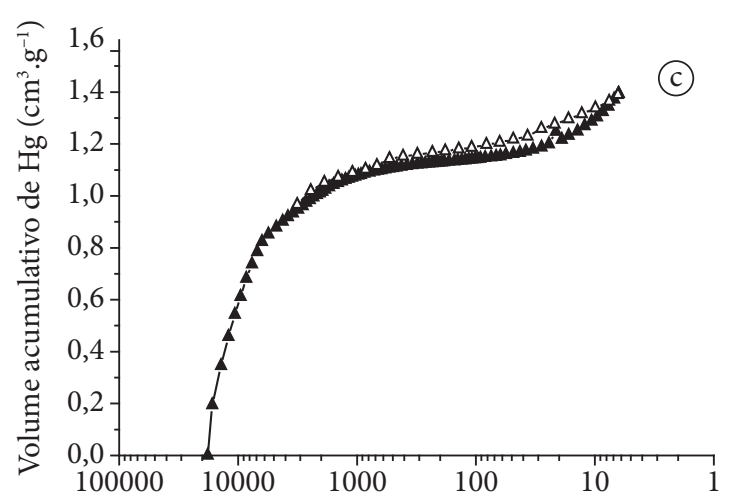

Diâmetro de poro (nm)

$$
-\Delta-\text { all3i }-\Delta-\text { all3e }
$$

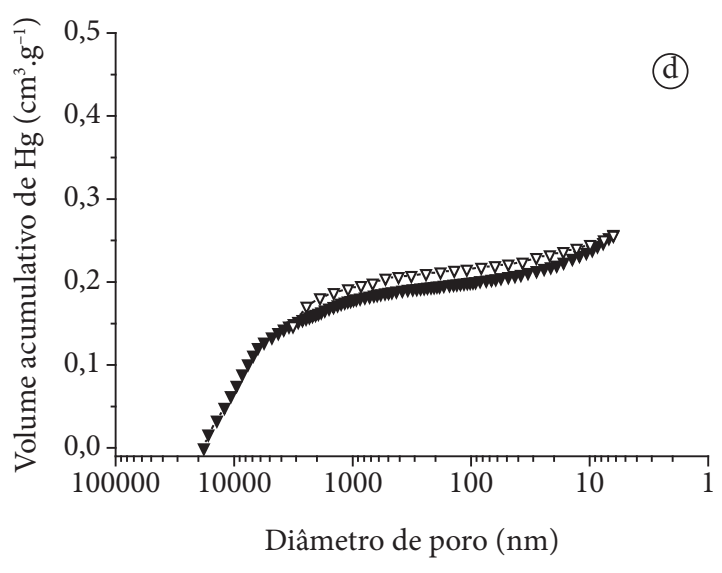

$$
\rightarrow \nabla-\text { als1i }-\nabla-\text { alsle }
$$

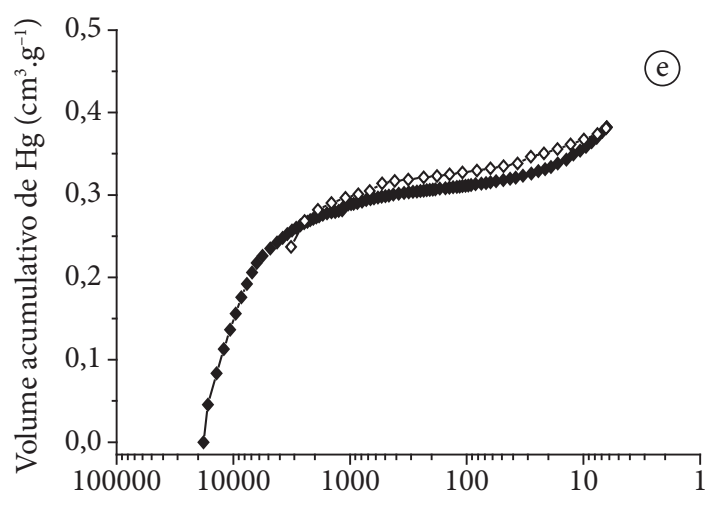

Diâmetro de poro (nm)

$$
\rightarrow \text { als } 2 \mathrm{i} \multimap \text { als2e }
$$

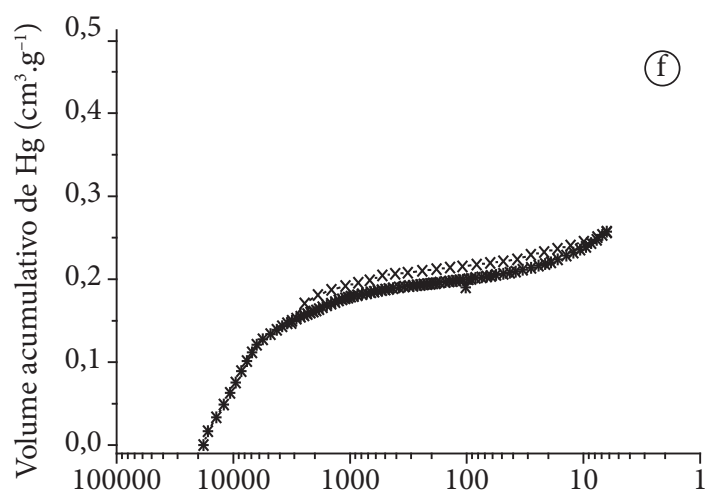

Diâmetro de poro (nm)

$$
\rightarrow \text { als } 3 \mathrm{i}-x-\text { als } 3 \mathrm{e}
$$

Figura 3. Curvas de intrusão (i) e extrusão (e) obtidas através da porosimetria de Mercúrio para fibra alimentar de albedo da laranja em diferentes intervalos granulométricos. a) Albedo de laranja liofilizado (0,42 a 0,30 mm); b) Albedo de laranja liofilizado (0,30 a 0,21 mm); c) Albedo de laranja liofilizado (0,21 a $0,14 \mathrm{~mm})$; d) Albedo de laranja secagem convencional (0,42 a 0,30 mm); e) Albedo de laranja secagem convencional (0,30 a 0,21 mm); e f) Albedo de laranja seco em bandeja (0,21 a 0,14 mm). 

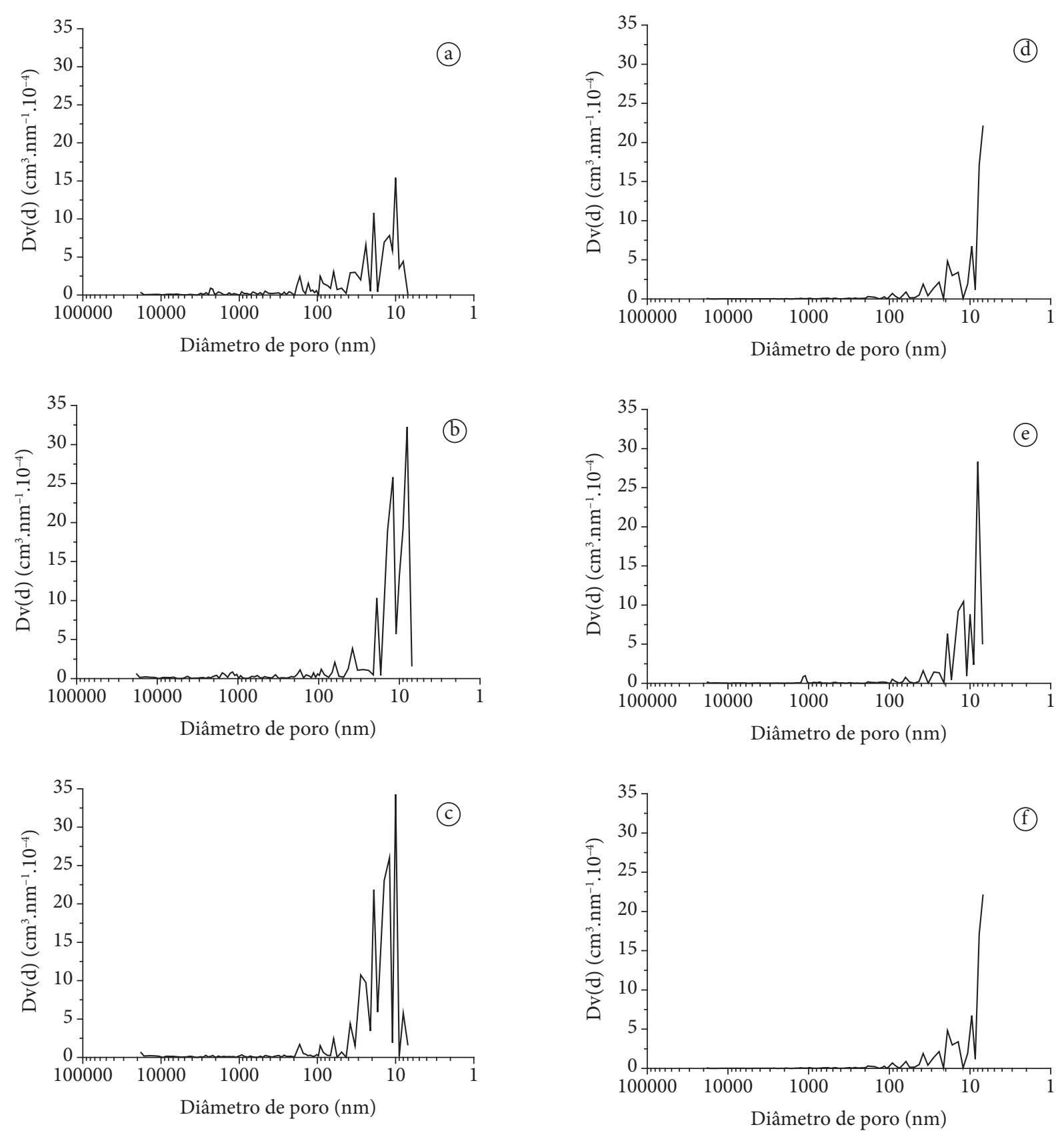

Figura 4. Distribuição de tamanho de poro de fibra alimentar de albedo de laranja por porosimetria de Mercúrio. a) Albedo de laranja liofilizado $(0,42$ a $0,30 \mathrm{~mm})$; b) Albedo de laranja liofilizado (0,30 a 0,21 mm); d) Albedo de laranja seco em secador convencional (042 a 0,30 mm); e e) Albedo de laranja seco em secador convencional (0,30 a $0,21 \mathrm{~mm})$.

Do ponto de vista microestrutural, é possível avaliar que o albedo de laranja possui grande potencial para enriquecer alimentos como fonte de fibra alimentar e que se fazem necessários estudos sobre os métodos de desidratação que lhes proporcionem melhores características ou menores danos físicos. Para tanto, as técnicas clássicas do sistema de tecnologia de partícula para determinação de parâmetros físicos das microestruturas de materiais orgânicos são viáveis sob condições de experimentação específicas.
A fibra alimentar de laranja apresentou características estruturais que permitem a diversificação de novos produtos alimentícios com alto valor nutritivo e comercial.

\section{Agradecimentos}

Os autores agradecem ao Laboratório Nacional de Luz Sincrotron (LNLS/MCT) pela disponibilidade do Microscópio Eletrônico de Varredura. 

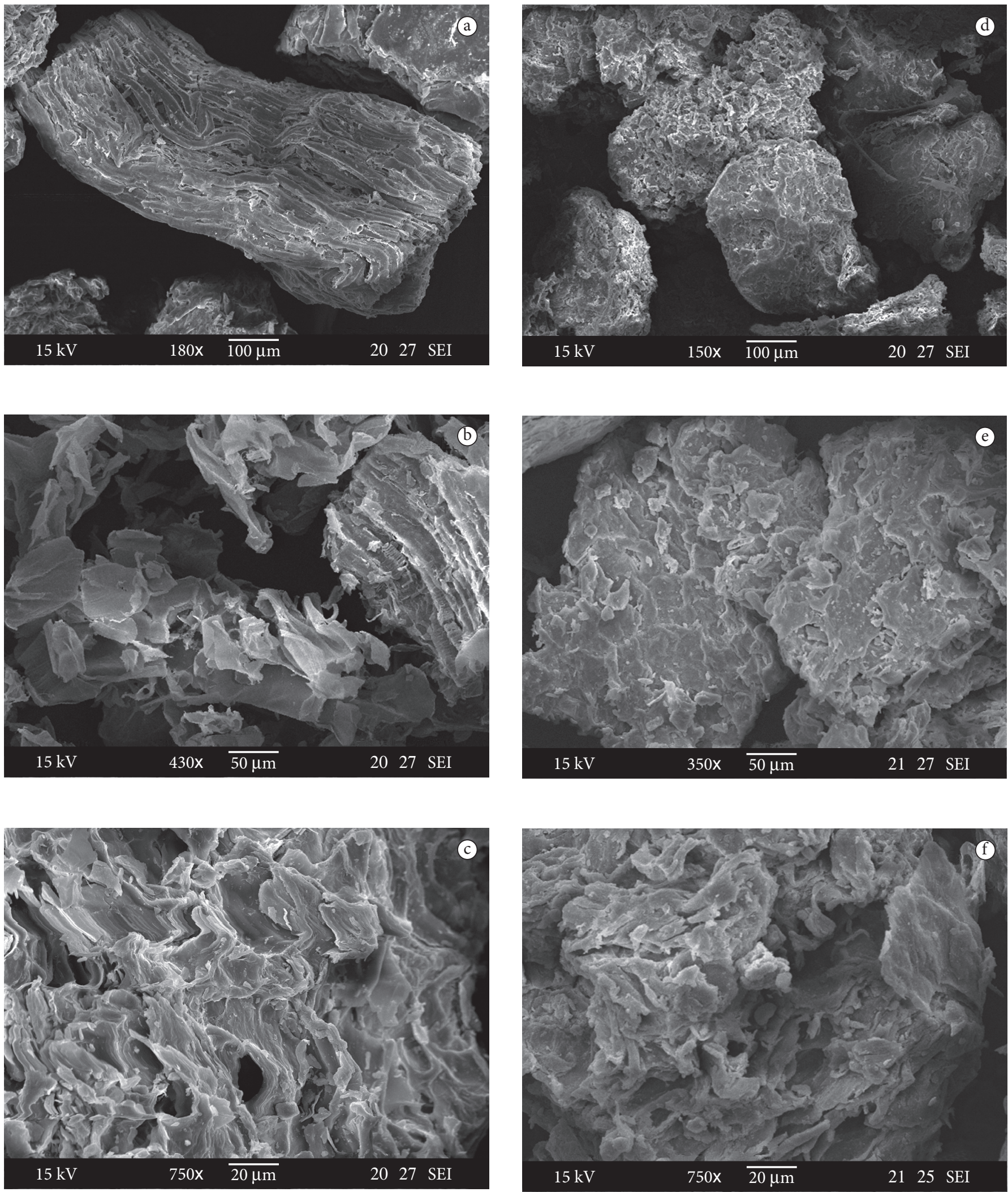

Figura 5. Microfotografias de fibra alimentar de albedo de laranja em diferentes métodos de desidratação e intervalos granulométricos. a) Albedo de Laranja Liofilizado T1 (180x); b) Albedo de Laranja Liofilizado T2 (430x); c) Albedo de Laranja Liofilizado T3 (750x); d) Albedo de Laranja Seco em em secador convencional T1 (150x); e) Albedo de Laranja Seco em secador convencional T2 (350x); e f) Albedo de Laranja Seco em secador convencional T3 (750x). 


\section{Referências Bibliográficas}

BARRET, E. P.; JOYNER, L. G.; HALENDA P. P. The determination opf pore volume and area distributions in porous substances Journal of American Chemistry Society, v. 73, n. 1, p. 373, 1951.

BERLIN, E.; HOWARD, N. M.; PALLANSCH, M. J. Specific surface áreas of milk powders produced by different drying methods. Journal of Dairy Science, v. 47, n. 2, p. 12-138, 1964.

BRUNAUER, S.; EMMETT, P. H.; TELLER, E. Adsorption of gases in multimolecular layers. Journal of American Chemistry Society, v. 60, p. 309-319, 1938.

CHESSON, A.; GARDNER, P. T.; WOOD, T. J. Cell wall porosity ad available surface area of wheat strw and wheat grain fractions. Journal of Science Food and Agriculture, v. 75, n. 3, p. 289-295, 1997.

COHAN, L. H. Hysteresis and the vapor pressure of concave surfaces. Journal of the American Chemical Society, v. 60, n. 2, p. 443-445, 1938.

COLLARES, F. P. Desprendimento de filmes de pastas alimentícias durante a secagem sobre superfícies de sólidos e sua relação com a transição vítrea. Campinas, 2002. 190p. Tese - (Doutorado em Tecnologia de Alimentos), Faculdade de Engenharia de Alimentos, Universidade Estadual de Campinas - UNICAMP.

IOANNIDIS, M.; CHARTIZ, I. A mixed-percolation model of capillary hysteresis and entrapment in mercury porosimetry. Journal of Colloid and Science, v. 161, n. 2, p. 278-291, 1993.

LARRAURI, J. A. New approaches in the preparation of high dietary fibre powders from fruit by-products. Food Science \& Technology, n. 10, p. 3-8, 1999.

LEE, K. H.; KIM, S. Y.; YOO, K. P. Evaluation of the poro-size distribuition in mercury porosimetry using computer simulations of porous media. Journal of Chemistry Engeneering, v. 11, n. 2, p. 131-135, 1994.

MATTHEWS, G. P.; RIDGWAY, C. J.; SPEARING, M. C. Void space modeling of mercury-intrusion hysteresis in sandstone, paper coating and other porous media. Journal of Colloid and Science, v. 171 , n. 1, p. 8-27, 1995.

PANCHEV, I.; KARAGEORGIEV, S. Investigations of some physical characteristics of plant structure which are used as sources of pectic substances. Internacional Journal of Food Science and Technology, v. 35, n. 3, p. 341-350, June, 2000.

ROUQUEROL, F.; ROUQUEROL, J.; SING, K. Adsorption by powders and porous solids: principles, methodology and applications. London: Academic press, 1999. 467p.

SCHOONMAN, A. et al. The microstructure of foamed maltodextrin/ sodium caseinate powders: a comparative study by microscopy and physical techniques. Food Research International, v. 34, p. 913-929, 2001.

SVAROVSKY, L. Powder Testing Guide Methods of Measuring the Physical Properties of Bulk Powders. London: British Materials Handling Board, 1987. $146 \mathrm{p}$.

WEBB, P. A.; ORR, C. Analytical methods in fine particle technology. Norcross: Micromeritics Instrument Corporation, 1997. 301 p.

WEBB, P. An introduction to the physical characterization of materials by mercury intrusion porosimetry with emphasis on reduction and presentation, 2001a. Disponível em: $<\mathrm{http}: / / \mathrm{www}$. micromeritics.com./pdf/app_articles/mercury_paper.pdf $>$ Acesso em: 17 Set. 2007

WEBB, P. A. Volume and Density Determinations for Particle Technologists, 2001b. Disponível em: <http://www.micromeritics. com/pdf/app_articles/density_determinations.pdf $>$. Acesso em: 17 Set. 2007. 